Review Article

\title{
Genetic Structure and Expression of the Surface Glycoprotein GP82, the Main Adhesin of Trypanosoma cruzi Metacyclic Trypomastigotes
}

\author{
Paulo Roberto Ceridorio Correa, Esteban Mauricio Cordero, Luciana Girotto Gentil, \\ Ethel Bayer-Santos, and José Franco da Silveira
}

\begin{abstract}
Departamento de Microbiologia, Imunologia e Parasitologia, Escola Paulista de Medicina, Universidade Federal de São Paulo, Rua Botucatu 862, 04023-062 São Paulo, SP, Brazil
\end{abstract}

Correspondence should be addressed to José Franco da Silveira; jose.franco@unifesp.br

Received 7 December 2012; Accepted 30 December 2012

Academic Editors: S. Amaral Gonçalves da Silva, P. M. L. Dutra, P. Grellier, and J. Martins de Santana

Copyright ( 2013 Paulo Roberto Ceridorio Correa et al. This is an open access article distributed under the Creative Commons Attribution License, which permits unrestricted use, distribution, and reproduction in any medium, provided the original work is properly cited.

T. cruzi improves the likelihood of invading or adapting to the host through its capacity to present a large repertoire of surface molecules. The metacyclic stage-specific surface glycoprotein GP82 has been implicated in host cell invasion. GP82 is encoded by multiple genes from the trans-sialidase superfamily. GP82 shows a modular organization, with some variation of N-terminal region flanking a conserved central core where the binding sites to the mammalian cell and gastric mucin are located. The function of GP82 as adhesin in host cell invasion process could expose the protein to an intense conservative and selective pressure. GP82 is a GPI-anchored surface protein, synthesized as a $70 \mathrm{kDa}$ precursor devoid of $N$-linked sugars. GPI-minus variants accumulate in the ER indicating that GPI anchor acts as a forward transport signal for progressing along the secretory pathway as suggested for T. cruzi mucins. It has been demonstrated that the expression of GP82 is constitutive and may be regulated at post-transcriptional level, for instance, at translational level and/or mRNA stabilization. GP82 mRNAs are mobilized to polysomes and consequently translated, but only in metacyclic trypomastigotes. Analysis of transgenic parasites indicates that the mechanism regulating GP82 expression involves multiple elements in the $3^{\prime}$ UTR.

\section{Introduction}

Trypanosoma cruzi is a protozoan parasite that causes Chagas disease, also called American trypanosomiasis, a debilitating and incurable disease affecting millions of people in Latin America. The life cycle of $T$. cruzi has multiple developmental stages: two in the invertebrate vector (triatomine hematophagous insects) and two in the vertebrate hosts. The infective forms are the trypomastigote stages found in the bloodstream of mammalian hosts and the metacyclic trypomastigotes present in the digestive tract of triatomines. Metacyclic trypomastigotes when eliminated in the feces of the triatomine can initiate the infection of mammalian hosts by invading a variety of cell types. They express a stagespecific surface glycoprotein of $82 \mathrm{kDa}$ (GP82) involved in host cell invasion that has no counterpart in bloodstream trypomastigotes [1-4]. GP82 is the major cell adhesion molecule of metacyclic forms that induces the activation of $\mathrm{Ca}^{+}$signaling cascades, leading to host cell cytoskeleton rearrangement and recruitment of lysosomes at the site of parasite entry, events required for the formation of the parasitophorous vacuole, and parasite internalization $[1,2,5-$ 8]. In this paper, we will mainly focus on the genetic structure of GP82 family and regulation of its expression by posttranscriptional control mechanisms.

\section{Structure of GP82 Gene}

The GP82 glycoprotein was first identified in the cellular surface of metacyclic forms by the monoclonal antibody Mab3F6 generated by immunization of mice with intact, 


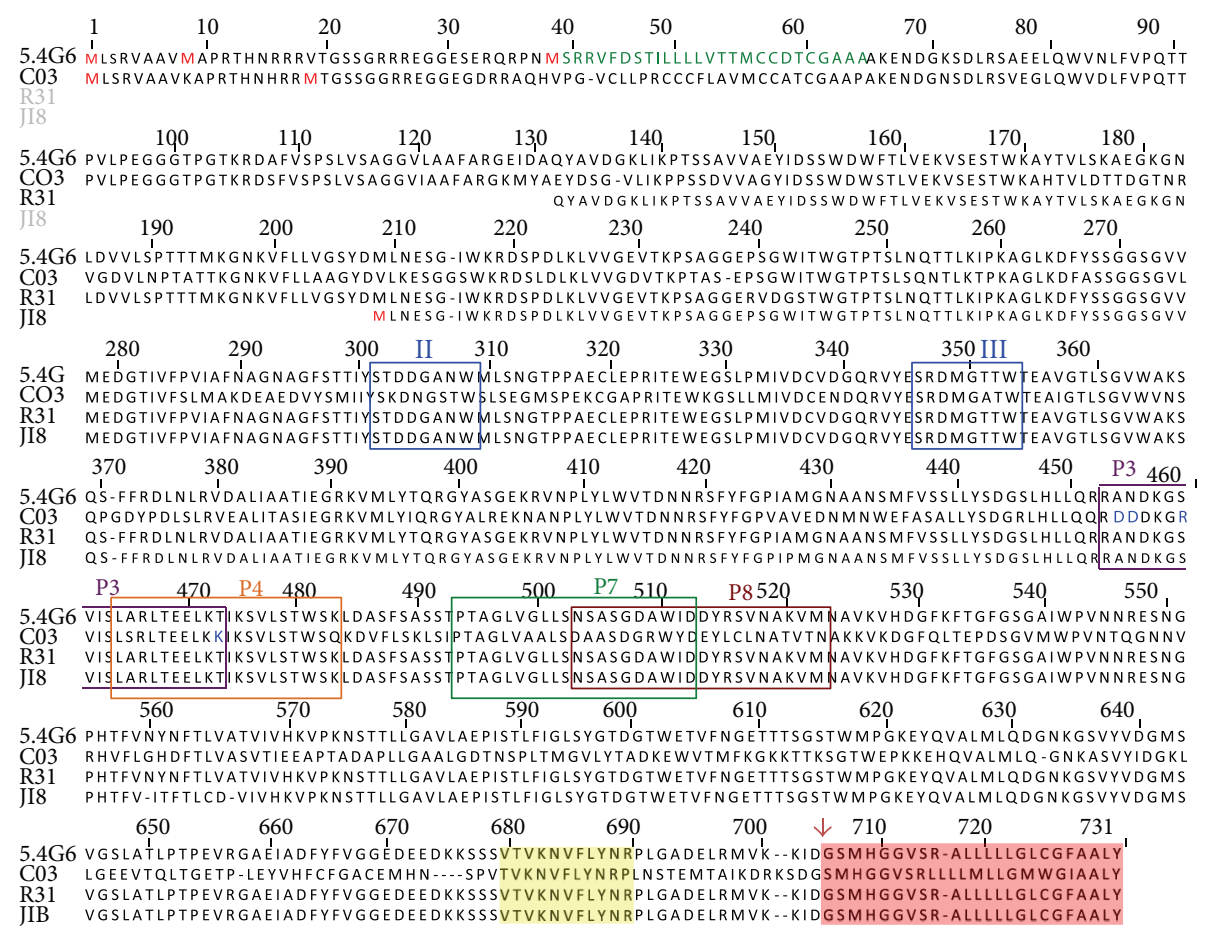

FIGURE 1: Alignment of amino acid sequences of some representatives of the GP82 family. Sequences are encoded by cDNA clones isolated from T. cruzi metacyclic trypomastigotes: 5.4G6 (ABR19835); CO3 (ABO28970); R31 (AF128843); J18 (AAA21303). GenBank accession numbers are in parentheses. Potential initiator methionines $(\mathrm{M})$ and a predicted $\mathrm{N}$-amino terminal signal peptide are indicated in red and green, respectively. The Asp boxes (bacterial sialidase motifs) are boxed and indicated by Roman numerals II and III. The epitope for Mab3F6 (P3), mammalian cell binding sites (P4 and P8), and gastric-mucin binding site (P7) are boxed and indicated by different colors. Note the overlapping between P3 and P4 sites, and P7 and P8 sites. The subterminal VTV motif, characteristic of the TS superfamily, and the potential GPI-anchor sequence are shaded in yellow and magenta, respectively. The arrow denotes the cleavage site for GPI anchor addition.

heat inactivated T. cruzi metacyclic forms [3, 4]. Since the determination of the first GP82 gene sequence in 1994 [9] many other sequences have become available [10-14], including those from T. cruzi genome sequencing projects [15-17]. The original analysis by Araya et al., 1994, showed the presence of two highly conserved Asp box domains (SxDxGxTW), previously described in bacterial sialidases, and a subterminal (VTVxNVFLYNR) motif (Figure 1) that are characteristics of the trans-sialidase (TS) superfamily of T. cruzi [18]. For this reason GP82 was classified in the TS superfamily $[9,18]$.

Figure 2 shows the comparison of five GP82 sequence variants isolated in our laboratory by cDNA cloning and three genomic sequences of clone CL Brener (T. cruzi genome project). Although all variants code for a glycosylphosphatidylinositol (GPI) anchor addition signal sequence at the carboxy-terminal (C-terminal), several of them do not have a signal peptide sequence at the amino-terminal $(\mathrm{N}-$ terminal), suggesting that they are not translocated into the endoplasmic reticulum (ER) and do not receive the GPI anchor.

Among the sequences annotated as GP82, the cDNA clone 5.4G6 (GenBank: EF154827) represents a complete transcript including the $5^{\prime}$ and $3^{\prime}$ untranslated regions (UTRs) (Figures 1 and 2) [12]. This clone encodes a protein of
726 amino acids (GenBank: ABR19835) that reacts with the Mab3F6 (Figure 1) [12]. The open reading frame (ORF) has three ATG initiation codons in the same reading frame, but only the third codon is inserted within the Kozak sequence context (Figure 2) [12]. It has been proposed that the Kozak sequence (consensus: GCCRCCaugG, $\mathrm{R}$ designates a purine base and aug the initiation codon), located upstream of the initiation codon, facilitates the mRNA translation [19]. Furthermore, clone 5.4G6 encodes an N-terminal signal peptide of 27 amino acids located just after the third methionine at position 39 and a signal sequence for cleavage/addition of GPI anchor at the C-terminal (Figures 1 and 2). Taking into account that the third ATG initiation codon follows the consensus Kozak sequence and the encoded protein has a predicted signal peptide, it is presumed that the translation initiates at the third methionine (Figure 2). The presence of 2-3 initiation codons in the same reading frame is relatively common among T. cruzi surface proteins, and after the second or the third methionine there is a sequence encoding a cryptic signal peptide as described in many genes as GP85 [20], Tc85 [21], ASP-2 [22], CRP-10 [23], GP90 [24], and SAP [25].

The existence of GP82 sequences lacking a typical Nterminal signal peptide suggests that its products may be located intracellularly, as occurs with the GP82 encoded by 


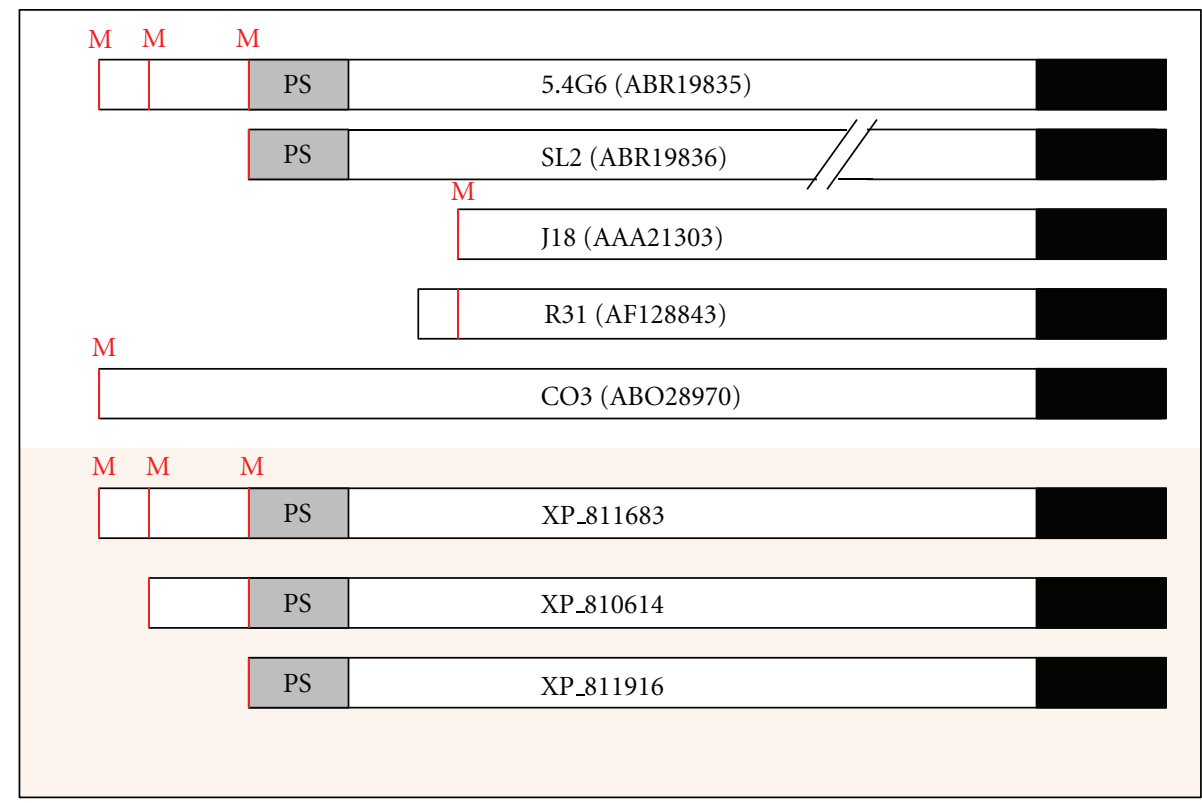

(a)

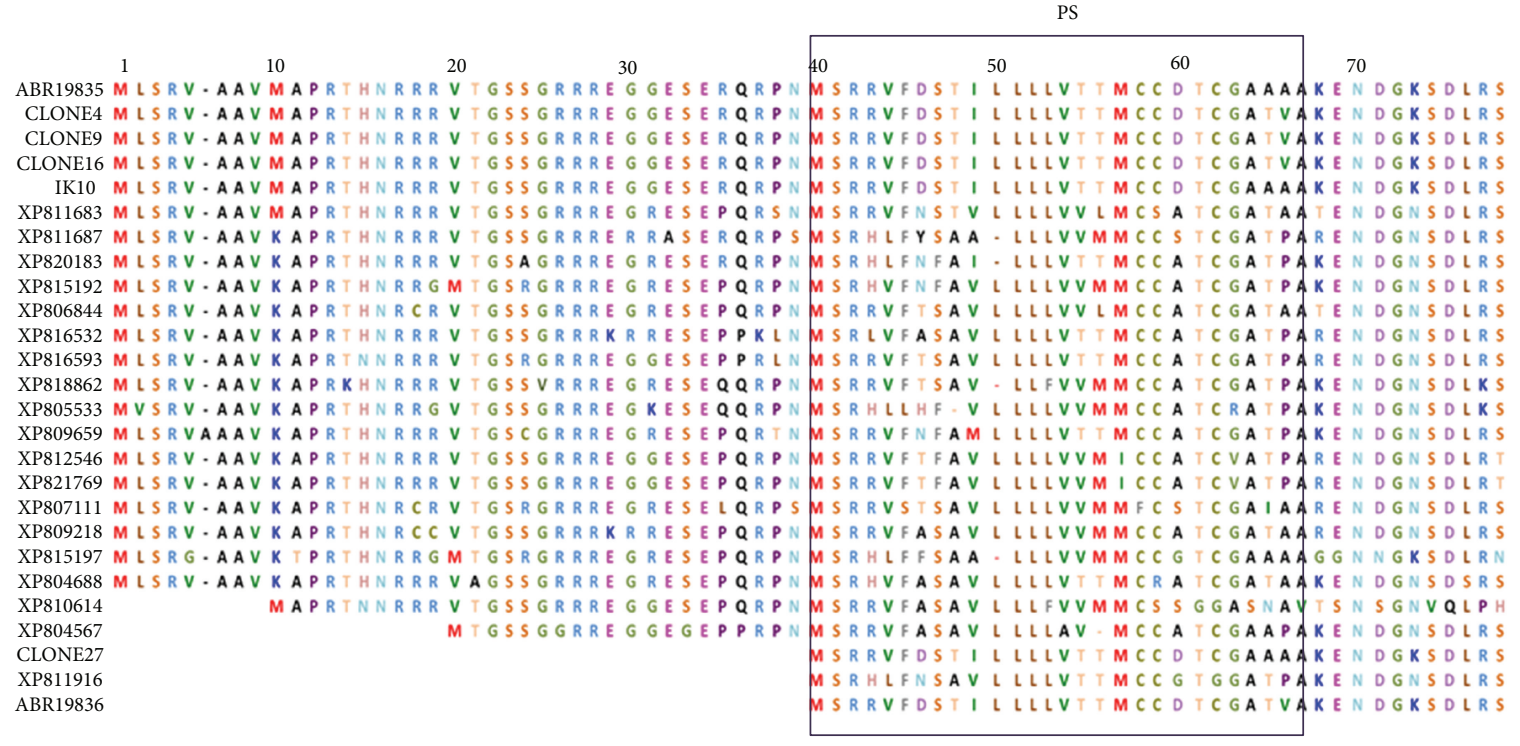

(b)

FIGURE 2: The modular architecture of GP82 family. (a) Structure of GP82 core proteins deduced from cDNA and genomic sequences. Sequences from cDNA clones are listed in the legend of Figure 1. SL (ABR19836) is a truncated cDNA sequence obtained by RT-PCR. The slashes indicate that sequence is interrupted. For ease of viewing, the putative C-terminal was drawn in the same line. GenBank accession numbers are in parentheses. The genomic sequences (GeneBank: XP_811683, XP_810614, XP_811916) were from the T. cruzi genome sequencing project (clone CL Brener). Potential initiator methionines (M), predicted $\mathrm{N}$-amino terminal signal peptide (SP), and potential GPI-anchor sequence are indicated in red, gray, and black, respectively. Not drawn to scale. (b) Alignment of GP82 sequences showing the variation of $\mathrm{N}$-terminal region. Potential initiator methionines (M) are indicated in red and the predicted $\mathrm{N}$-amino terminal signal peptide (PS) is boxed. cDNA sequences: ABR19835, clones 4, 9, 16, IK10 and 27, ABR19835, ABR19836; genomic sequences were extracted from the T. cruzi genome sequencing project (clone CL Brener) and are indicated by the prefix XP..

the clone C03 (Figure 1) which is located at the flagellum of metacyclic forms of the CL strain [13]. Such GP82 protein displaying flagellar localization is not involved in the invasion of mammalian cells by metacyclic forms [13]. Data obtained with monospecific anti-GP82 antibodies support the hypothesis that GP82 proteins that have no N-terminal signal peptide are located intracellularly and are not involved in host cell invasion [13].

The members of TS superfamily show a highly conserved hydrophobic sequence (M S R R V F/T S V L L L L F/L 
$\mathrm{V}$ ) at the $\mathrm{N}$-terminal region, which acts as a signal peptide addressing the nascent protein into the ER. Analysis of the N-terminal region of the CRP-10 from TS superfamily indicates that this sequence functions as signal peptide [23]. Based on the analysis of GP82 variants characterized to date, we could suggest that metacyclic trypomastigotes simultaneously express different variants of GP82, and their cellular localization is determined by the $\mathrm{N}$-terminal signal peptide. However, as the N-terminal of native GP82 has not been determined, we cannot rule out the possibility that the translation starts in different ATG codons.

The epitope recognized by Mab3F6 and the site of adhesion of GP82 to the host cells were identified by incubating recombinant proteins and synthetic peptides in mammalian in vitro invasion assays. Regions of GP82 gene coding for the C-terminal, central, and $\mathrm{N}$-terminal domains were subcloned into plasmid pGEX and expressed in E. coli. The reactivity with Mab3F6 and the ability of each recombinant protein to inhibit cell invasion were tested [6], both the Mab3F6 (P3) binding site and the host cell adhesion sites (P4 and P8) were identified in the central domain of GP82 (Figure 1) as will be discussed in the topic below.

\section{Organization of GP82 Gene Family}

T. cruzi genome comprises more than $50 \%$ of repetitive sequences including several multigene families that encode surface proteins. Among them, the most abundant is the TS superfamily [17]. The genome sequencing of clone CL Brener [17] confirmed the complexity of the TS superfamily by identification of 1,430 sequences, including 737 genes and 693 pseudogenes. These sequences have been annotated as transsialidase in the T. cruzi genome project with no mention to which group or family they could be included.

According to sequence identity, molecular weight, and function, members of TS superfamily were classified into four groups or families [18, 26-29]. Members of a family or group exhibit $\geq 60 \%$ similarity among each other, whereas similarity among members of different families or groups may vary from 20 to $40 \%$. Group I of the TS superfamily comprises proteins with enzymatic activity; that is, they are enzymes (trans-sialidase) able to transfer sialic acid from a donor to the mucins present at $T$. cruzi surface $[18,26-29]$.

The members of group II were also called "trans-sialidase like" proteins because they have no enzymatic activity [18, 26-29]. These proteins have complete or degenerate Asp box motifs (SxDxGxTW), the VTVxNVFLYNR motif characteristic of all TS members, and the signal sequence for cleavage/addition of GPI anchor at the C-terminal region [18, 26-29]. Group II comprises the surface glycoproteins GP85, Tc85, TSA-1, SA85, GP90, GP82, ASP-1, and ASP-2 which are involved in adhesion and invasion of mammalian cells $[18,20-22,24,26-30]$. Several proteins of this group are also targets of the host immune system and may induce protective immunity in animal models [30]. The proteins of group III (CRP, FL160, CEA, and TESA) inhibit the classical and alternative pathways of complement activation and are recognized by sera from patients with Chagas disease $[18$,
26-29, 31, 32]. Group IV is composed of genes encoding trypomastigote surface antigens that have no defined biological function $[18,26,27]$.

Recently, Freitas et al., 2011, [33] reported an extensive and detailed analysis of TS sequences of clone CL Brener that resulted in the redistribution of members in 8 different groups designated as TcSgroupI to TcSgroupVIII. The sequences analyzed in this study $(n=508)$ were categorized according to structure, function, presence of conserved motifs, chromosomal localization, expression profiling, and antigenic properties. TcSgroupI to TcSgroupIV $(n=176)$ correspond to groups I to IV described above. There is a good correlation with the classification proposed previously $[18,26,27]$ and with the prior annotation made in our laboratory [16]. The new classification proposed by Freitas et al., 2011, [33] could categorize 329 sequences that were included in the groups TcSgroupV-TcSgroupVIII.

To identify the repertoire of GP82 genes in the genome of clone CL Brener (the reference clone of T. cruzi Genome Project), we carried out a BLASTP search using the GP82 encoded by clone 5.4G6 (GenBank: ABR19835) as query [10]. We identified 19 complete sequences with $>60 \%$ identity with the query which were considered as GP82 and distributed as follows: 2 proteins (GenBank: XP_811663 and XP_804688) with $70-81 \%$ identity and the remaining with $61-68 \%$ identity (see Figure 2). Pseudogenes and truncated sequences were discarded from the analysis. Although GP82 are encoded from a relatively small number of genes, the repertoire is quite variable. This contrasts with other TS-like protein families which are composed of large sets of genes such as GP85, Tc85, GP90, and ASP [16, 20, 21, 24, 33].

The ability of genes to be robust to mutations at the codon level has been suggested as a key factor for understanding adaptation features. It has been proposed that genes relevant to host-parasite interactions will tend to exhibit high volatility or "anti-robust" patterns, which may be related to the parasite capacity of evading the host immune system [34]. We investigated the potential capacity of $T$. cruzi surface protein genes to maximize phenotypic variation, which may be seen as a key attribute to expand the repertoire of surface antigens [34]. The robustness of a parasite gene against mutations was addressed in terms of several gene volatility and diversity indicators. The potential impact of point-mutation errors on surface antigen genes based on the analysis of codon usage and its potential for generating different amino acid mutants were explored. These data were consistent with the low rate of volatility calculated using the GP82 sequences deposited in GenBank [16]. GP82 genes have "low volatility" which means that the mutations are generally synonymous or lead replacing amino acids with others of the same polarity. GP82 genes seem to be genetically "robust"; that is, they exhibit a tendency to neutralize the mutations encoding the same amino acid or an amino acid of the same polarity.

Analysis of selective pressure on GP82 variants showed that the protein could have undergone conservative or negative selection. The function of GP82 as adhesin in host cell invasion process could expose the protein to an intense conservative and selective pressure. The potential variability 
of GP82 genes suggests that they are "robust" or are not susceptible to mutation [16].

In the human protozoan parasites Trypanosoma brucei and Plasmodium falciparum, the subtelomeric regions play an important role in generation of new variants of surface antigen genes and in the control of gene expression [3537]. We reported that $T$. cruzi subtelomeric regions are enriched in (pseudo)genes from the TS superfamily, DGF-1, and retrotransposon hot spot protein (RHS) families [38, 39]. The abundance of surface protein genes in the subtelomeric regions suggests that these regions may have acted as a site for DNA recombination, expansion, and the generation of new variants of surface proteins. Moraes Barros et al. (2012) [39] demonstrated that all the groups of the TS superfamily are represented in the subtelomeric regions of clone CL Brener; most of the sequences $(n=83)$ are members of group II (GP82, GP85, TC85), which includes 22 complete genes. It is interesting to note that 7 out of 19 GP82 genes identified in clone CL Brener are located at subtelomeric regions.

\section{Synthesis and Processing of GP82}

GP82 is attached to the outer parasite's cell membrane by a GPI anchor $[3,40]$. It is synthesized as a $70 \mathrm{kDa}$ precursor devoid of $N$-linked sugars and when mature, it has an apparent molecular weight of $82 \mathrm{kDa}$. GP82 binds to the target cell in a dose-dependent and saturable fashion and reduces the infection of Vero cells by metacyclic forms of CL and Tulahuen strains by 90 to 97 and $50 \%$, respectively [8].

The immunological screening of a metacyclic cDNA library with the Mab3F6 allowed the isolation of a 2,140 bp clone, named J18 (GenBank L14824), which encodes a protein of 516 amino acids containing the functional domains of GP82 [9]. Analysis of the deduced amino acid sequence showed the presence of three sialidase domains (two conserved and one slightly degenerated), a VTV motif, four putative $\mathrm{N}$-glycosylation sites, and a GPI-anchor addition signal, which allows us to classify the GP82 in group II of TS superfamily [9].

Recombinant expression of clone J18 and a series of step-wise deletions enabled the identification of the domain involved in the adhesion to the mammalian cells and indirectly, the region containing the epitope for Mab3F6 [6]. A central region spanning 132 amino acids was identified as the responsible for the adhesin properties and ten overlapping peptides encompassing this central domain were synthesized to further characterize the region. The authors found two non-contiguous peptides with significant adhesive properties, named P4 and P8; thus, they speculated about a putative conformational binding-domain in the native protein, in which these two peptides would be in close proximity [6].

To further address the adhesin activity of these peptides (P4 and P8) and to rule out any peptide's solubility and conformational artifacts, both peptides were expressed in a non-adherent microorganism. The expression of GP82 cell binding peptides $\mathrm{P} 4$ or P8 in the fourth surface-exposed loop of the transmembrane protein LamB of Escherichia coli conferred the ability to this microorganism to adhere to the surface of HeLa cells [41]. Between the two populations of bacteria, those carrying the $\mathrm{P} 4$ peptide were almost twice more efficient to adhere to HeLa cells than the population expressing the P8. In the same way, the expression of GP82 protein on the outer membrane of non-infective T. cruzi epimastigotes enabled these non-adherent forms to attach to the surface of HeLa cells [42].

A more detailed analysis on the central domain of GP82 was performed by Manque et al., 2000, [43] using the same peptides described above by Santori et al., 1996, [6] and variants of GP82 lacking the regions corresponding to the peptides P4 and P8. This strategy allowed the authors to identify the peptide P3 as the epitope recognized by Mab3F6. As the peptide P3 has ten amino acids overlapping with the peptide P4 (cell binding site), this finding provided support for the inhibition of parasite's invasion by Mab3F6, which is probably due to sterical hindrance. Additionally, the authors were able to confirm the GP82 conformational cell-binding domain hypothesis raised by Santori et al., 1996, [6] by means of the hybrid peptide P4/8 which contained 17 amino acids from P4 and 5 amino acids from P8 peptide. This peptide $\mathrm{P} 4 / 8$ was more efficient than $\mathrm{P} 4$ and $\mathrm{P} 8$ peptides to inhibit the binding of the recombinant GP82 to the HeLa cells [43].

Recently, it was demonstrated that GP82 binds specifically to gastric mucin in the oral infection $[44,45]$. The implication of GP82 in adhesion of metacyclic forms to the gastric mucin was first described by Neira et al., 2003, [45] and further confirmed by Staquicini et al., 2010, [44] using a GP82 recombinant protein Del-4/8 lacking the central domain of the molecule. The GP82 binding to the gastric mucin may direct the adhesion and invasion of the stomach epithelium by the metacyclic forms. Tests of invasion inhibition of the gastric mucosa showed that peptide P7 (Figure 1), located in the central domain of the molecule, contains the binding site to the gastric mucin [44]. The in vitro inhibitory effect of peptide P7 was reproducible in vivo in murine model [44].

Experimental evidence of the GP82 GPI-anchor was given by Cardoso De Almeida \& Heise (1993) [40] through digestion with phosphatidylinositol-specific phospholipase $\mathrm{C}$ (PI-PLC) and phase separation in Triton X-114. Araya et al., 1994, [9] predicted the putative GP82 GPI-anchor cleavage/addition site based on the sequence of the clone J18. Similarly, Ramirez et al., 1999, [14, 46] analyzed in more detail the GPI-anchor signal of GP82 and other T. cruzi proteins and conducting homologous and heterologous expressions of GP82 in T. cruzi epimastigotes and mammalian cell systems. Despite the absence of a typical signal peptide in the protein encoded by the J18 clone, the authors found that T. cruzi machinery was able to translocate the protein inside the ER finally deliver it to the parasite's cell surface (Ramirez et al., 1999) [14]. On the other hand, when the same protein was synthesized by mammalian cells, it failed to translocate inside the ER and accumulated in the cytoplasm, indicating the requirement for a typical signal peptide. When the required signal was provided by insertion of the signal peptide from the influenza virus hemagglutinin, the mammalian cell was able to translocate the GP82 chimera inside the ER but proved insufficient to provide expression on the cell surface. These 
findings indicated that the requirements for GPI-anchoring are different between T. cruzi and mammalian cells [14, 46].

In order to further dissect the requirements for GPIanchoring between mammals and T. cruzi, a site-directed mutagenesis was performed in the GPI cleavage/addition signal [47]. The putative GPI-anchor acceptor domain determined by Ramirez et al., 1999, [46] is formed by the amino acids aspartic $(\omega)$, glycine $(\omega+1)$, and serine $(\omega+2)$ (DGS) where the aspartic acid is linked to the GPI-anchor. A single mutation was introduced changing the aspartic acid to serine generating the sequence (SGS) which previously proved to be a feasible signal for GPI anchoring of T. brucei Variant Surface Glycoprotein (VSG) in mammals [48]. An additional construct lacking the GPI-anchor was created and transfected either in mammalian cells or T. cruzi epimastigotes [47].

Confocal analyses on transfected parasites showed that the point mutation had no detectable effect on the GPIanchoring efficiency [47]. The deletion of the GPI-signal resulted in a protein that was not anchored but accumulated in the parasite cytoplasm instead [47]. These findings were in agreement with those obtained in T. brucei by Böhme and Cross (2002) [49] where the parasite was able to anchor several mutated proteins but not those in which the GPIanchor signal was deleted. On the other hand, the mammalian cells failed to express all the transfected proteins on the cell surface, even the point mutation which proved to be functional for GPI anchoring in mammals [48].

Isolation of GPI-anchored proteins can be accomplished by digestion with enzymes that cleave specifically this structure as glycosylphosphatidylinositol-specific phospholipase C (GPI-PLC) or phosphatidylinositol-specific phospholipase $\mathrm{C}$ (PI-PLC). This very simple approach can be hindered by the presence of acylation in position 6 of the inositol ring (sometimes the acylation occurs at position 5) due to steric hindrance.

Due to the presence of the GPI anchor, proteins carrying this modification acquire an overall hydrophobic behavior. Based on this property, detergents can be used to concentrate/enrich fractions in this particular kind of proteins by temperature-induced partition. Bordier 1981 [50] first described the suitability of the detergent Triton X-114 (TX114) to concentrate hydrophobic proteins due to its nearly physiological clouding point temperature. At temperatures above $23^{\circ} \mathrm{C}$ a formerly homogenous solution containing the detergent TX-114 will split into two phases: an upper layer depleted of detergent (hydrophilic) and a lower phase enriched in detergent's micelles (hydrophobic). Using this physical property, Cordero et al., 2009, [51] concentrated the GPI-anchored proteins of metacyclic and epimastigotes of T. cruzi after several consecutive partitions in TX-114. Mass spectrometry analyses on those fractions detected several members of TS superfamily, among those, the surface glycoprotein GP82 had 22\% of its sequence covered by tryptic peptides. Among those peptides, the Asp boxes, VTV, and cell binding site P8 were mapped [51]. Other important region mapped in this study was peptide P7, which was identified as the binding site for the gastric mucin [44].

Several proteomics studies have been conducted in metacyclic forms of T. cruzi, but to the best of our knowledge, there is only one additional report of GP82 elsewhere [52]. Recently, a quantitative proteomic study was performed in parasites undergoing metacyclogenesis [53]. Among the proteins identified in this study, authors found 38 members of TS superfamily. Due to lack of a unified/standardized annotation among the databases and the absence of the peptide sequences used in this study, it was not possible to determine the presence of GP82 among them. Some of those annotated TSs shared a high degree of identity with GP82 protein, but because of the missing peptide sequences it is impossible to assign an unambiguous classification. The lack of a common nonredundant annotation represents an issue that must be taken in consideration with an urge to amend.

Recently, Cortez et al., 2012, [54] compiled all the biochemical, physicochemical, and functional information available on GP82 in order to create the most updated model of the protein structure. The authors based this model on the homology of T. cruzi GP82 (GenBank: L14824) with T. rangeli sialidase (PDB 1N1T_A), a close related molecule which had its crystallographic structure (inhibitor-bound) already solved. The sketched GP82 appears as two clearly different and separated domains (an amino-terminal $\beta$-propeller and a $\beta$-sandwich C-terminal domain) linked together by an $\alpha$ helix. In this layout, P3, P4, P7, and P8 motifs have a variable degree of access to the solvent. The cell-binding peptide P4 encompasses $2 / 3$ of the $\alpha$-helix that bridges the protein together and is fully exposed. On the other hand, peptide P8 located in the carboxy-terminal domain, although exposed, has limited solvent accessibility. The partial exposure of the P8 motif complies with the experimental data and gives a topological explanation for the limited role of P8 in the GP82 binding to the cell [41]. As expected, the P3 motif containing the epitope for the 3F6 antibody was fully exposed and accessible to the solvent, reinforcing the Mab3F6 inhibitory effect by steric hindrance. The gastric mucin-binding motif P7 was poorly exposed, mostly due to its high hydrophobicity. The amino-terminal residues of this motif (P7) are completely buried, leaving just the C-terminal portion partially exposed, mainly because it overlaps with the P8 motif. In summary, this model seems to fulfill the requirements for structural analysis and provides an appropriated support to the biological experimental data.

\section{Post-Transcriptional Control Mechanisms of GP82 Expression}

In trypanosomes, post-translational control mechanisms play an important role in gene expression regulation due to unique features related to transcription, mRNA maturation, and stabilization over the parasite life cycle. T. cruzi genome is organized in large gene clusters separated by divergent strand-switch regions [55], and transcription of these clusters produces large primary transcripts that are processed by trans-splicing and polyadenylation to generate mature mRNAs [56]. These processes are guided by pyrimidinerich regions contained in the polycistronic transcripts [57, 58]. Although there is no transcription regulation in $T$. cruzi, proteomic analysis of the four developmental stages 
(epimastigote, metacyclic trypomastigote, amastigote, and bloodstream trypomastigote) demonstrated that there was a significant change in relative protein abundance throughout life cycle [52]. Furthermore, microarray analysis showed that at least $50 \%$ of T. cruzi genes are regulated during its life cycle [59]. GP82 is one of these differentially regulated proteins, and the mechanisms regulating its stage-specific expression began to be clarified.

Steady-state levels of GP82 transcripts from T. cruzi G strain were determined by northern blot, dot-blot hybridization, and quantitative real-time PCR, demonstrating that there is a significant increase in GP82 mRNA levels in metacyclic forms when compared with the other three stages $[9,11,60]$. Northern blot analysis revealed a single band of $2.2 \mathrm{~kb}$ mRNA only in metacyclic forms [9]. Dot-blot hybridization showed that GP82 transcript levels were around 5.5-fold higher in metacyclic trypomastigotes than in other stages [60]. Similar results were obtained using quantitative real-time PCR (unpublished data). Moreover, expression analysis of other three GP82 gene subfamilies from Peru2 strain, called groups $\mathrm{A}, \mathrm{B}$, and $\mathrm{C}$, showed an increase in mRNA accumulation (4.7 to 9.3-fold) in metacyclic forms when compared to epimastigotes [11]. Additionally, GP82 protein stage-specific expression was also showed by western blot using the Mab3F6 [3]. Even though GP82 mRNA and protein were barely detected in epimastigotes, nuclear runon analysis demonstrated that GP82 gene was transcribed in both epimastigote and metacyclic forms, confirming that transcript accumulation in metacyclic forms is not due to an increased transcription rate, but rather to some posttranslational control [60].

Changes in GP82 mRNA stability were detected and thought to be responsible for differences in its steady-state level. Parasites treated with actinomycin D had their GP82 transcript half-lives estimated to be about $6 \mathrm{~h}$ in metacyclic forms and $0.5 \mathrm{~h}$ in epimastigotes [60]. Cycloheximide treatment increased GP82 levels in epimastigotes, suggesting that a labile protein factor was responsible for destabilizing mRNA in these forms and prevent mRNA translation. In addition, GP82 mRNAs were only found associated with polysomes in metacyclic forms [60], indicating that transcript mobilization to polysomes might be involved in regulating GP82 expression, as was reported for another T. cruzi gene [61].

There are at least three known factors that modulate mRNA steady-state level: cis-acting elements, transacting factors, and the apparatus involved in mRNA turnover and degradation [62]. Cis-acting elements are noncoding sequences that act from inside the same molecule (intramolecular action). Trans-acting factors are diffuse molecules, usually proteins, that act from a different molecule to regulate a target mRNA (intermolecular action) [63]. The fate of transcripts is determined by the interaction of cisacting sequences present in the $3^{\prime}$ UTR with specific transacting protein factors containing RNA-binding domains that subsequently recruit the protein machinery to destroy or stabilize mRNAs [64]. The involvement of GP82 $3^{\prime}$ UTR in mRNA stability was analyzed using a reporter green fluorescent protein (GFP) fused upstream to the GP82 $3^{\prime}$ UTR.
Parasites transfected with an episomal plasmid carrying this construct had their GFP protein and mRNA levels analyzed, revealing that the $3^{\prime}$ UTR was able to downregulate GFP in epimastigotes and upregulate it in metacyclic forms [65]. Similar mechanisms for controlling mRNA stability by $3^{\prime}$ UTR sequences have also been described for other TS family members, such as the flagellum-associated surface protein FL-160 (TcS group III) [66], two genes coding for active trans-sialidase enzymes from TcS group I, described by Jager et al., 2008, [67], and another TS member [64]. There are pieces of evidence that stem-loop secondary structures formed in the $3^{\prime}$ UTR might be responsible for the interaction with RNA-binding proteins [68]. Prediction of GP82 $3^{\prime}$ UTR secondary structure was performed in silico using mfold program [69], revealing the presence of stem-loop structures; however, the role of these structures was not analyzed so far.

Regulatory cis-acting elements of variable sizes were identified in the $3^{\prime}$ UTR of some trypanosomatid genes (reviewed in $[62,70]$ ). In the case of GP82, four step-wise deletions were performed to search for regulatory elements in its $3^{\prime}$ UTR. Results indicated that more than one region was responsible for changing GFP mRNA and protein levels in epimastigotes and metacyclic forms [65], suggesting that multiple cis-acting elements are present in GP82 $3^{\prime}$ UTR and might bind to distinct RNA-binding proteins (RBP). The first trans-acting factor identified in T. cruzi was TcUBP1 (T. cruzi uridine binding protein 1), which binds to AU-rich elements of the TcSMUG mRNA leading to its destabilization [71]. In addition to TcSMUG, other 39 transcripts were found bound to TcUBP1 by co-immunoprecipitation assays [68]. One common cis-acting element was identified in the $3^{\prime}$ UTRs of the majority of these TcUBP1 target mRNAs. This ciselement was used to predict novel UBP1 target mRNAs and GP82 was one of them [68]. Therefore, TcUBP1 could be one of the trans-acting factors involved in GP82 mRNA stability. A schematic representation of the mechanism controlling GP82 gene expression is shown in Figure 3.

There are growing pieces of evidence suggesting the presence of post-transcriptional operons in trypanosomes, mediated by the coordinated interaction between cis-elements and trans-acting factors $[68,72]$. It was demonstrated that a group of $T$. brucei stage-regulated proteins share a specific sequence motif in the $3^{\prime}$ UTR (reviewed in [72]). Also, two RBPs from T. cruzi, TcUBP1, and TcUBP3, preferentially associate with a set of functionally related transcripts bearing the same RNA motif that is recognized by each protein [68]. These post-transcriptional operons could explain how coordinately expression regulation is achieved in organisms where genespecific transcriptional control is absent.

\section{Concluding Remarks and Perspectives}

GP82 shows a modular organization, with some variation of $\mathrm{N}$-terminal region flanking a conserved central core where the binding sites to mammalian cell and gastric mucin are located. The function of GP82 as adhesin in host cell invasion process could expose the protein to an intense conservative and selective pressure. The potential variability of GP82 


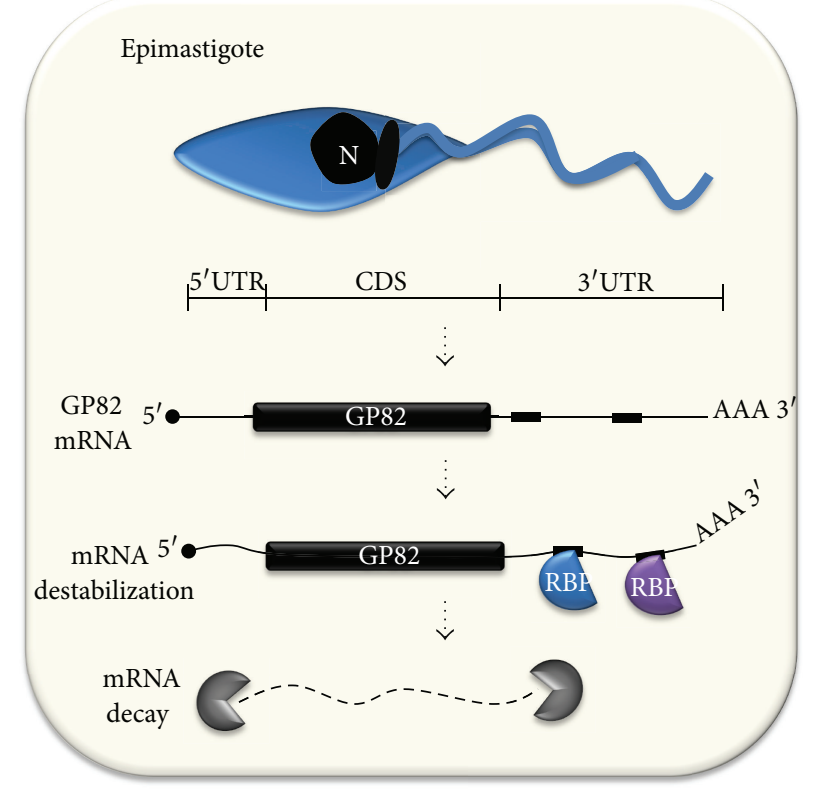

(a)

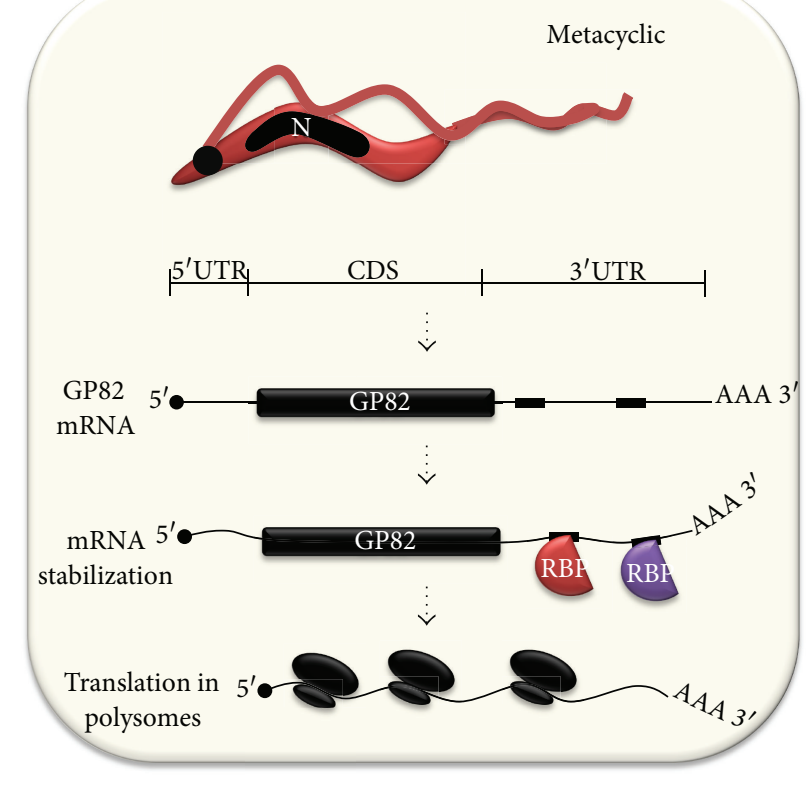

(b)

FIGURE 3: Comparison of GP82 mRNA post-transcriptional control mechanisms in (a) epimastigotes and (b) metacyclic trypomastigotes. In epimastigotes, GP82 mRNA interacts with possibly more than one RNA-binding protein (RBP), which binds to different cis-elements in the $3^{\prime}$ UTR region (small black rectangles), leading to mRNA destabilization and decay. Conversely, in the metacyclic trypomastigote stage, a different set of RBPs interacts with the cis-elements present in the $3^{\prime} \mathrm{UTR}$, promoting mRNA stabilization and translation in polysomes.

genes suggests that they are not susceptible to mutation. The many isoforms of GP82 and its multiple N-terminal variants suggest that some GP82 family members might display different cellular localizations and functions. The challenge is to ascertain the relationships between GP82 gene sequences, protein isoforms, and its distinct or overlapping functions.

GP82 is a GPI-anchored surface protein, synthesized as a $70 \mathrm{kDa}$ precursor devoid of $\mathrm{N}$-linked sugars and when mature has an apparent molecular weight of $82 \mathrm{kDa}$. GPI-minus variants accumulate in the ER indicating that GPI anchor acts as a forward transport signal for progressing along the secretory pathway as suggested for T. cruzi mucins [73]. Heterologous expression of GP82 into mammalian cells indicated that the requirements for GPI-anchoring are different between $T$. cruzi and mammalian cells. These differences could be targets for the development of parasite-specific therapeutic agents.

Several studies demonstrated that the transcription of GP82 is constitutive and may be regulated at posttranscriptional level, for instance, at translational level and/or mRNA stabilization. GP82 mRNAs are mobilized to polysomes and consequently translated, but only in metacyclic trypomastigotes. It has been suggested that the stabilizing mechanism acting in metacyclic trypomastigotes and the destabilizing mechanism in epimastigotes could be mediated by a cis-acting element present in the $3^{\prime}$ UTR of transcripts. A series of step-wise deletions in the $3^{\prime}$ UTR was created and results suggest that the mechanism regulating GP82 expression involves multiple elements in the $3^{\prime}$ UTR. Interestingly, the $3^{\prime}$ UTR of GP82 transcript promotes higher expression of the green fluorescent protein (GFP) reporter in metacyclic trypomastigotes than in epimastigotes.

In conclusion, while our knowledge of the structure and function of GP82 is large, there still remain many questions to be answered. Additional studies are carried out to analyze the expression, localization, and involvement in host cell invasion of each GP82 variant identified to date.

\section{Acknowledgments}

This work was supported by grants from FAPESP and CNPq (Brazil) to J. F. da Silveira. P. R. C. Correa and E. Bayer-Santos are awarded a doctoral fellowship from FAPESP and CNPq, Brazil, respectively.

\section{References}

[1] N. Yoshida, K. M. Tyler, and M. S. Llewellyn, "Invasion mechanisms among emerging food-borne protozoan parasites," Trends in Parasitology, vol. 27, no. 10, pp. 459-466, 2011.

[2] N. Yoshida, "Molecular basis of mammalian cell invasion by Trypanosoma cruzi," Anais da Academia Brasileira de Ciencias, vol. 78, no. 1, pp. 87-111, 2006.

[3] M. M. G. Teixeira and N. Yoshida, "Stage-specific surface antigens of metacyclic trypomastigotes of Trypanosoma cruzi identified by monoclonal antibodies," Molecular and Biochemical Parasitology, vol. 18, no. 3, pp. 271-282, 1986.

[4] N. Yoshida, "Surface antigens of metacyclic trypomastigotes of Trypanosoma cruzi, Infection and Immunity, vol. 40, no. 2, pp. 836-839, 1983. 
[5] R. C. Ruiz, S. Favoreto, M. L. Dorta et al., "Infectivity of Trypanosoma cruzi strains is associated with differential expression of surface glycoproteins with differential $\mathrm{Ca}^{2+}$ signalling activity," Biochemical Journal, vol. 330, part 1, pp. 505-511, 1998.

[6] F. R. Santori, M. L. Dorta, L. Juliano et al., "Identification of a domain of Trypanosoma cruzi metacyclic trypomastigote surface molecule gp82 required for attachment and invasion of mammalian cells," Molecular and Biochemical Parasitology, vol. 78, no. 1-2, pp. 209-216, 1996.

[7] M. L. Dorta, A. T. Ferreira, M. E. M. Oshiro, and N. Yoshida, " $\mathrm{Ca}^{2+}$ signal induced by Trypanosoma cruzi metacyclic trypomastigote surface molecules implicated in mammalian cell invasion," Molecular and Biochemical Parasitology, vol. 73, no. 1-2, pp. 285-289, 1995.

[8] M. I. Ramirez, R. De Cassia Ruiz, J. E. Araya, J. F. Da Silveira, and N. Yoshida, "Involvement of the stage-specific 82kilodalton adhesion molecule of Trypanosoma cruzi metacyclic trypomastigotes in host cell invasion," Infection and Immunity, vol. 61, no. 9, pp. 3636-3641, 1993.

[9] J. E. Araya, M. I. Cano, N. Yoshida, and J. F. Da Silveira, "Cloning and characterization of a gene for the stage-specific $82-\mathrm{kDa}$ surface antigen of metacyclic trypomastigotes of Trypanosoma cruzi," Molecular and Biochemical Parasitology, vol. 65, no. 1, pp. 161-169, 1994.

[10] P. R. C. Correa, Study of genetic variability of the surface antigen GP82 of metacyclic trypomastigotes of Trypanosoma cruzi: identification of transcripts repertoire and the bindingsite to the mammalian cell [Ph.D. Doctoral Thesis], Federal University of São Paulo (UNIFESP), São Paulo, Brazil, 2012.

[11] D. Songthamwat, K. Kajihara, M. Kikuchi et al., "Structure and expression of three gp82 gene subfamilies of Trypanosoma cruzi," Parasitology International, vol. 56, no. 4, pp. 273-280, 2007.

[12] E. M. Cordero, In vitro and in vivo expression of genes encoding for surface antigens GP82 and GP90 of metacyclic trypomastigotes of Trypanosoma cruzi [Ph.D. Doctoral Thesis], Federal University of São Paulo (UNIFESP), São Paulo, Brazil, 2012.

[13] V. D. Atayde, M. Cortez, R. Souza, J. F. Da Silveira, and N. Yoshida, "Expression and cellular localization of molecules of the gp82 family in Trypanosoma cruzi metacyclic trypomastigotes," Infection and Immunity, vol. 75, no. 7, pp. 3264-3270, 2007.

[14] M. I. Ramirez, S. B. Boscardin, S. W. Han et al., "Heterologous expression of a Trypanosoma cruzi surface glycoprotein (gp82) in mammalian cells indicates the existence of different signal sequence requirements and processing," Journal of Eukaryotic Microbiology, vol. 46, no. 6, pp. 557-565, 1999.

[15] O. Franzen, E. Arner, M. Ferella et al., "The short non-coding transcriptome of the protozoan parasite Trypanosoma cruzi," PLoS Neglected Tropical Diseases, vol. 5, no. 8, Article ID e1283, 2011.

[16] F. Azuaje, J. L. Ramirez, and J. F. Da Silveira, "An exploration of the genetic robustness landscape of surface protein families in the human protozoan parasite Trypanosoma cruzi," IEEE Transactions on Nanobioscience, vol. 6, no. 3, pp. 223-228, 2007.

[17] N. M. El-Sayed, P. J. Myler, D. C. Bartholomeu et al., "The genome sequence of Trypanosoma cruzi, etiologic agent of chagas disease," Science, vol. 309, no. 5733, pp. 409-435, 2005.

[18] W. Colli, "Trans-sialidase: a unique enzyme activity discovered in the protozoan Trypanosoma cruzi," The FASEB Journal, vol. 7, no. 13, pp. 1257-1264, 1993.
[19] M. Kozak, "Effects of intercistronic length on the efficiency of reinitiation by eucaryotic ribosomes," Molecular and Cellular Biology, vol. 7, no. 10, pp. 3438-3445, 1987.

[20] G. B. Takle and G. A. M. Cross, "An 85-kilodalton surface antigen gene family of Trypanosoma cruzi encodes polypeptides homologous to bacterial neuraminidases," Molecular and Biochemical Parasitology, vol. 48, no. 2, pp. 185-198, 1991.

[21] R. Giordano, D. L. Fouts, D. Tewari, W. Colli, J. E. Manning, and M. J. M. Alves, "Cloning of a surface membrane glycoprotein specific for the infective form of Trypanosoma cruzi having adhesive properties to laminin," The Journal of Biological Chemistry, vol. 274, no. 6, pp. 3461-3468, 1999.

[22] H. P. Low and R. L. Tarleton, "Molecular cloning of the gene encoding the $83 \mathrm{kDa}$ amastigote surface protein and its identification as a member of the Trypanosoma cruzi sialidase superfamily," Molecular and Biochemical Parasitology, vol. 88, no. 1-2, pp. 137-149, 1997.

[23] K. A. Norris, J. E. Schrimpf, and M. J. Szabo, "Identification of the gene family encoding the 160-kilodalton Trypanosoma cruzi complement regulatory protein," Infection and Immunity, vol. 65, no. 2, pp. 349-357, 1997.

[24] M. S. Do Carmo, M. R. M. Dos Santos, M. I. Cano, J. E. Araya, N. Yoshida, and J. F. Da Silveira, "Expression and genomewide distribution of the gene family encoding a $90 \mathrm{kDa}$ surface glycoprotein of metacyclic trypomastigotes of Trypanosoma cruzi," Molecular and Biochemical Parasitology, vol. 125, no. 1-2, pp. 201-206, 2002.

[25] R. C. P. Baida, M. R. M. Santos, M. S. Carmo et al., "Molecular characterization of serine-, alanine-, and proline-rich proteins of Trypanosoma cruzi and their possible role in host cell infection," Infection and Immunity, vol. 74, no. 3, pp. 1537-1546, 2006.

[26] O. Campetella, D. Sanchez, J. J. Cazzulo, and A. C. C. Frasch, "A superfamily of Trypanosoma cruzi surface antigens," Parasitology Today, vol. 8, no. 11, pp. 378-381, 1992.

[27] S. Schenkman, D. Eichinger, M. E. A. Pereira, and V. Nussenzweig, "Structural and functional properties of Trypanosoma trans-sialidase," Annual Review of Microbiology, vol. 48, pp. 499-523, 1994.

[28] G. A. M. Cross and G. B. Takle, "The surface trans-sialidase family of Trypanosoma cruzi," Annual Review of Microbiology, vol. 47, pp. 385-411, 1993.

[29] A. C. C. Frasch, "Functional diversity in the trans-sialidase and mucin families in Trypanosoma cruzi," Parasitology Today, vol. 16, no. 7, pp. 282-286, 2000.

[30] C. Claser, N. M. Espíndola, G. Sasso, A. J. Vaz, S. B. Boscardin, and M. M. Rodrigues, "Immunologically relevant strain polymorphism in the Amastigote Surface Protein 2 of Trypanosoma cruzi," Microbes and Infection, vol. 9, no. 8, pp. 1011-1019, 2007.

[31] M. Beucher and K. A. Norris, "Sequence diversity of the Trypanosoma cruzi complement regulatory protein family," Infection and Immunity, vol. 76, no. 2, pp. 750-758, 2008.

[32] T. K. Matsumoto, P. C. Cotrim, J. F. Da Silveira, A. M. S. Stolf, and E. S. Umezawa, "Trypanosoma cruzi: isolation of an immunodominant peptide of TESA (trypomastigote excretedsecreted antigens) by gene cloning," Diagnostic Microbiology and Infectious Disease, vol. 42, no. 3, pp. 187-192, 2002.

[33] L. M. Freitas, S. L. dos Santos, G. F. Rodrigues-Luiz et al., "Genomic analyses, gene expression and antigenic profile of the trans-sialidase superfamily of Trypanosoma cruzi reveal an undetected level of complexity," PLoS One, vol. 6, no. 10, Article ID e25914, 2011. 
[34] J. B. Plotkin, J. Dushoff, and H. B. Fraser, "Detecting selection using a single genome sequence of $M$. tuberculosis and $P$. falciparum," Nature, vol. 428, no. 6986, pp. 942-945, 2004.

[35] L. H. Freitas-Junior, E. Bottius, L. A. Pirrit et al., "Frequent ectopic recombination of virulence factor genes in telomeric chromosome clusters of P. falciparum," Nature, vol. 407, no. 6807, pp. 1018-1022, 2000.

[36] C. B. B. Lira, M. A. Giardini, J. L. S. Neto, F. F. Conte, and M. I. N. Cano, "Telomere biology of trypanosomatids: beginning to answer some questions," Trends in Parasitology, vol. 23, no. 8, pp. 357-362, 2007.

[37] D. Horn and J. D. Barry, "The central roles of telomeres and subtelomeres in antigenic variation in African trypanosomes," Chromosome Research, vol. 13, no. 5, pp. 525-533, 2005.

[38] D. Kim, M. A. Chiurillo, N. El-Sayed et al., "Telomere and subtelomere of Trypanosoma cruzi chromosomes are enriched in (pseudo)genes of retrotransposon hot spot and trans-sialidaselike gene families: the origins of T. cruzi telomeres," Gene, vol. 346, pp. 153-161, 2005.

[39] R. Moraes Barros, M. Marini, C. Antonio et al., "Anatomy and evolution of telomeric and subtelomeric regions in the human protozoan parasite Trypanosoma cruzi," BMC Genomics, vol. 13, article 229, 2012.

[40] M. L. Cardoso De Almeida and N. Heise, "Proteins anchored via glycosylphosphatidylinositol and solubilizing phospholipases in Trypanosoma cruzi," Biological Research, vol. 26, no. 1-2, pp. 285-312, 1993.

[41] C. M. Pereira, S. Favoreto, J. F. Da Silveira, N. Yoshida, and B. A. Castilho, "Adhesion of Escherichia coli to HeLa cells mediated by Trypanosoma cruzi surface glycoprotein-derived peptides inserted in the outer membrane protein LamB," Infection and Immunity, vol. 67, no. 9, pp. 4908-4911, 1999.

[42] P. M. Manque, I. Neira, V. D. Atayde et al., "Cell adhesion and $\mathrm{Ca}^{2+}$ signaling activity in stably transfected Trypanosoma cruzi epimastigotes expressing the metacyclic stage-specific surface molecule gp82," Infection and Immunity, vol. 71, no. 3, pp. 15611565, 2003.

[43] P. M. Manque, D. Eichinger, M. A. Juliano, L. Juliano, J. E. Araya, and N. Yoshida, "Characterization of the cell adhesion site of Trypanosoma cruzi metacyclic stage surface glycoprotein gp82," Infection and Immunity, vol. 68, no. 2, pp. 478-484, 2000.

[44] D. I. Staquicini, R. M. Martins, S. Macedo et al., "Role of GP82 in the selective binding to gastric mucin during oral infection with Trypanosoma cruzi," PLoS Neglected Tropical Diseases, vol. 4, no. 3, Article ID e613, 2010.

[45] I. Neira, F. A. Silva, M. Cortez, and N. Yoshida, "Involvement of Trypanosoma cruzi metacyclic trypomastigote surface molecule gp82 in adhesion to gastric mucin and invasion of epithelial cells," Infection and Immunity, vol. 71, no. 1, pp. 557-561, 2003.

[46] M. I. Ramirez, S. B. Boscardin, R. C. Ruiz et al., "Heterologous expression of a Trypanosoma cruzi surface glycoprotein (gp82) indicates that requirements for glycosylphosphatidylinositol anchoring are different in mammalian cells and this trypanosome," Memórias do Instituto Oswaldo Cruz, vol. 94, no. 4, pp. 527-530, 1999.

[47] E. M. Cordero, Study of processing surface glycoprotein of $82 \mathrm{kDa}$ (GP82) of metacyclic trypomastigotes of Trypanosoma cruzi [M.Sc. thesis], Federal University of São Paulo (UNIFESP), São Paulo, Brazil, 2002.

[48] P. Moran and I. W. Caras, "Requirements for glycosylphosphatidylinositol attachment are similar but not identical in mammalian cells and parasitic protozoa," Journal of Cell Biology, vol. 125, no. 2, pp. 333-343, 1994.

[49] U. Böhme and G. A. M. Cross, "Mutational analysis of the variant surface glycoprotein GPI-anchor signal sequence in Trypanosoma brucei," Journal of Cell Science, vol. 115, no. 4, pp. 805-816, 2002.

[50] C. Bordier, "Phase separation of integral membrane proteins in Triton X-114 solution," The Journal of Biological Chemistry, vol. 256, no. 4, pp. 1604-1607, 1981.

[51] E. M. Cordero, E. S. Nakayasu, L. G. Gentil, N. Yoshida, I. C. Almeida, and J. F. Da Silveira, "Proteomic analysis of detergentsolubilized membrane proteins from insect-developmental forms of Trypanosoma cruzi," Journal of Proteome Research, vol. 8, no. 7, pp. 3642-3652, 2009.

[52] J. A. Atwood, D. B. Weatherly, T. A. Minning et al., "Microbiology: the Trypanosoma cruzi proteome," Science, vol. 309, no. 5733, pp. 473-476, 2005.

[53] L. M. de Godoy, F. K. Marchini, D. P. Pavoni et al., "Quantitative proteomics of Trypanosoma cruzi during metacyclogenesis," Proteomics, vol. 12, no. 17, pp. 2694-2703, 2012.

[54] C. Cortez, N. Yoshida, D. Bahia, and T. Sobreira, "Structural basis of the interaction of a Trypanosoma cruzi surface molecule implicated in oral infection with host cells and gastric mucin," PLoS One, vol. 7, no. 7, Article ID e42153, 2012.

[55] S. Martínez-Calvillo, J. C. Vizuet-de-Rueda, L. E. FlorencioMartínez, R. G. Manning-Cela, and E. E. Figueroa-Angulo, "Gene expression in trypanosomatid parasites," Journal of Biomedicine and Biotechnology, vol. 2010, Article ID 525241, 15 pages, 2010.

[56] X. H. Liang, A. Haritan, S. Uliel, and S. Michaeli, "trans and cis splicing in trypanosomatids: mechanism, factors, and regulation," Eukaryotic Cell, vol. 2, no. 5, pp. 830-840, 2003.

[57] C. Hartmann, H. R. Hotz, M. McAndrew, and C. Clayton, "Effect of multiple downstream splice sites on polyadenylation in Trypanosoma brucei," Molecular and Biochemical Parasitology, vol. 93, no. 1, pp. 149-152, 1998.

[58] C. Lopez-Estrano, C. Tschudi, and E. Ullu, "Exonic sequences in the $5^{\prime}$ untranslated region of $\alpha$-tubulin mRNA modulate trans splicing in Trypanosoma brucei," Molecular and Cellular Biology, vol. 18, no. 8, pp. 4620-4628, 1998.

[59] T. A. Minning, D. B. Weatherly, J. Atwood, R. Orlando, and R. L. Tarleton, "The steady-state transcriptome of the four major life-cycle stages of Trypanosoma cruzi," BMC Genomics, vol. 10, article 370, 2009.

[60] L. G. Gentil, E. M. Cordero, M. S. D. Carmo, M. R. M. D. Santos, and J. F. D. Silveira, "Posttranscriptional mechanisms involved in the control of expression of the stage-specific GP82 surface glycoprotein in Trypanosoma cruzi," Acta Tropica, vol. 109, no. 2, pp. 152-158, 2009.

[61] A. R. Avila, S. F. Yamada-Ogatta, V. Da Silva Monteiro et al., "Cloning and characterization of the metacyclogenin gene, which is specifically expressed during Trypanosoma cruzi metacyclogenesis," Molecular and Biochemical Parasitology, vol. 117, no. 2, pp. 169-177, 2001.

[62] C. Clayton and M. Shapira, "Post-transcriptional regulation of gene expression in trypanosomes and leishmanias," Molecular and Biochemical Parasitology, vol. 156, no. 2, pp. 93-101, 2007.

[63] J. G. De Gaudenzi, G. Noe, V. A. Campo, A. C. Frasch, and A. Cassola, "Gene expression regulation in trypanosomatids," Essays in Biochemistry, vol. 51, pp. 31-46, 2011. 
[64] P. R. Araujo, G. A. Burle-Caldas, R. A. Silva-Pereira, D. C. Bartholomeu, W. D. daRocha, and S. M. R. Teixeira, "Development of a dual reporter system to identify regulatory cisacting elements in untranslated regions of Trypanosoma cruzi mRNAs," Parasitology International, vol. 60, no. 2, pp. 161-169, 2011.

[65] E. Bayer-Santos, L. G. Gentil, E. M. Cordero, P. R. Correa, and J. F. da Silveira, "Regulatory elements in the $3^{\prime}$ untranslated region of the GP82 glycoprotein are responsible for its stage-specific expression in Trypanosoma cruzi metacyclic trypomastigotes," Acta Tropica, vol. 123, no. 3, pp. 230-233, 2012.

[66] D. Weston, A. C. La Flamme, and W. C. Van Voorhis, "Expression of Trypanosoma cruzi surface antigen FL-160 is controlled by elements in the $3^{\prime}$ untranslated, the $3^{\prime}$ intergenic, and the coding regions," Molecular and Biochemical Parasitology, vol. 102, no. 1, pp. 53-66, 1999.

[67] A. V. Jager, R. P. Muia, and O. Campetella, "Stage-specific expression of Trypanosoma cruzi trans-sialidase involves highly conserved 3' untranslated regions," FEMS Microbiology Letters, vol. 283, no. 2, pp. 182-188, 2008.

[68] G. Noe, J. G. De Gaudenzi, and A. C. Frasch, "Functionally related transcripts have common RNA motifs for specific RNAbinding proteins in trypanosomes," BMC Molecular Biology, vol. 9, article 107, 2008.

[69] M. Zuker, "Mfold web server for nucleic acid folding and hybridization prediction," Nucleic Acids Research, vol. 31, no. 13, pp. 3406-3415, 2003.

[70] S. Haile and B. Papadopoulou, "Developmental regulation of gene expression in trypanosomatid parasitic protozoa," Current Opinion in Microbiology, vol. 10, no. 6, pp. 569-577, 2007.

[71] I. D’Orso and A. C. C. Frasch, “TcUBP-1, a developmentally regulated U-rich RNA-binding protein involved in selective mRNA destabilization in trypanosomes," The Journal of Biological Chemistry, vol. 276, no. 37, pp. 34801-34809, 2001.

[72] S. Kramer and M. Carrington, “Trans-acting proteins regulating mRNA maturation, stability and translation in trypanosomatids," Trends in Parasitology, vol. 27, no. 1, pp. 23-30, 2011.

[73] G. E. Canepa, A. C. Mesias, H. Yu, X. Chen, and C. A. Buscaglia, "Structural features affecting trafficking, processing, and secretion of Trypanosoma cruzi mucins," The Journal of Biological Chemistry, vol. 287, no. 31, pp. 26365-26376, 2012. 

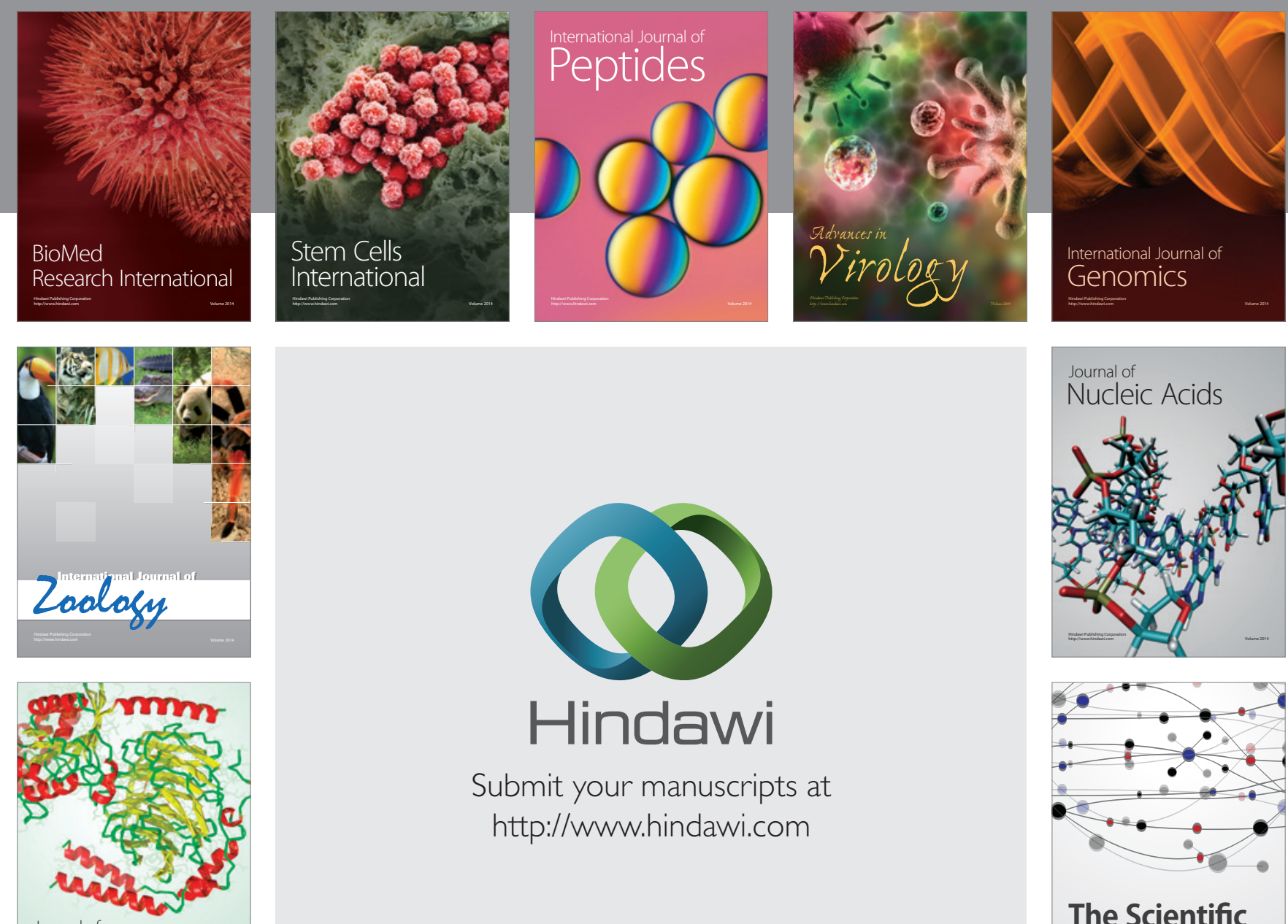

Submit your manuscripts at

http://www.hindawi.com

Journal of
Signal Transduction
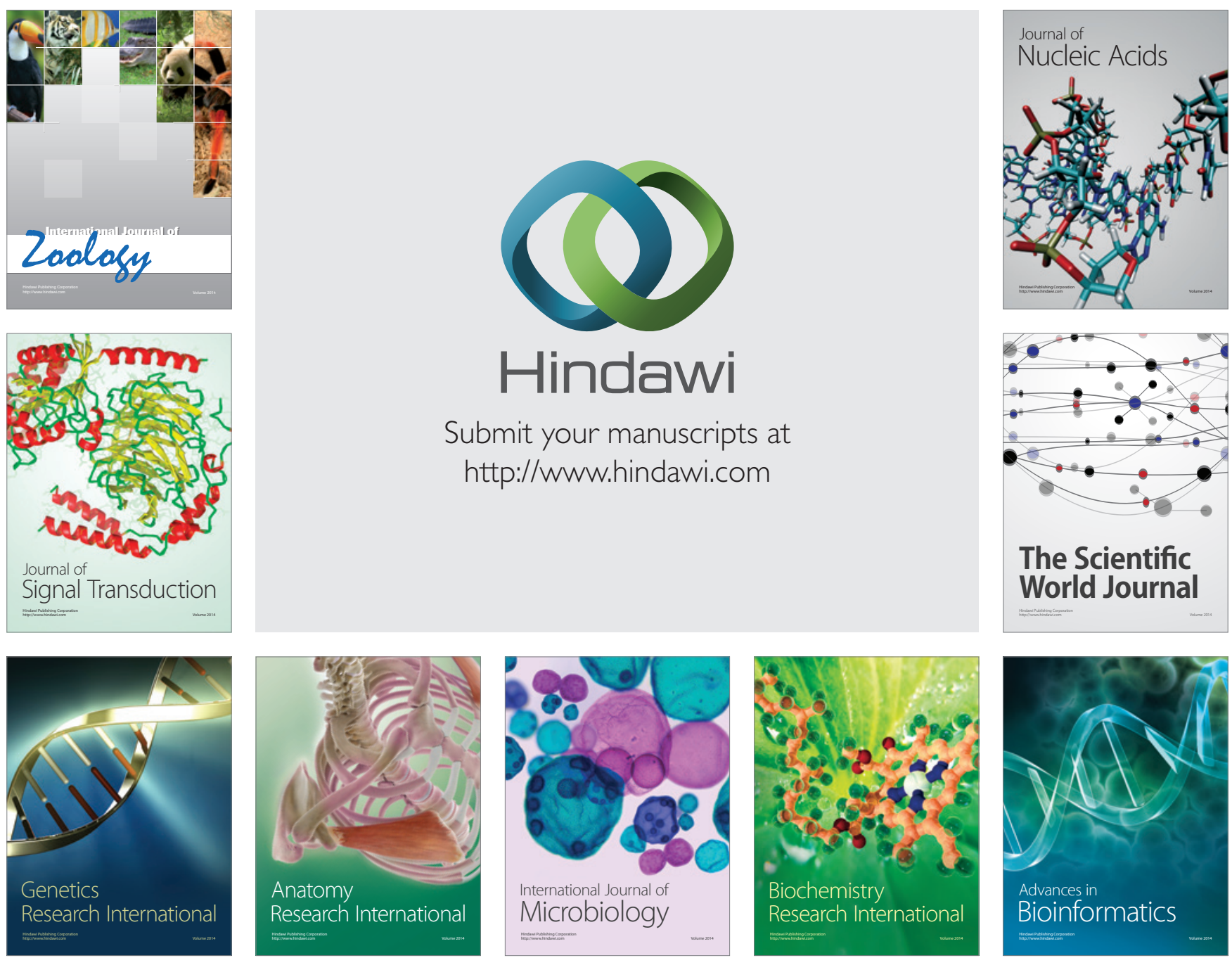

The Scientific World Journal
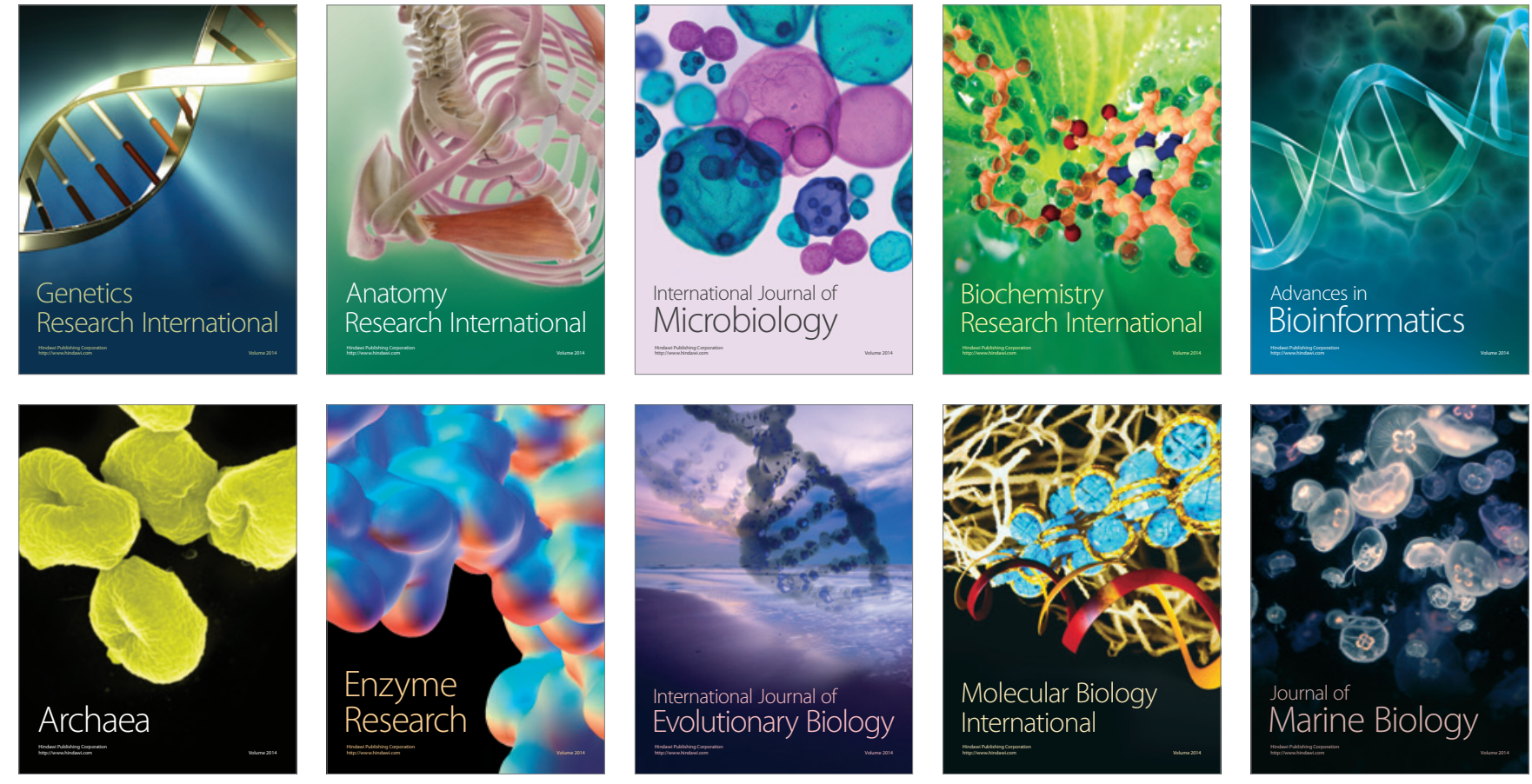